\title{
Cerebellopontine Angle Epidermoid Cysts: Surgical Treatment Outcome
}

\author{
Mohamed I. Refaat ${ }^{1}$ Omar Y. Abdallah ${ }^{1}$ \\ ${ }^{1}$ Department of Neurosurgery, School of Medicine, \\ Cairo University, Egypt
}

\begin{abstract}
Address for correspondence Mohamed I. Refaat, PhD, Department of Neurosurgery, Cairo University Hospitals, Elmanial, Cairo 11562, Egypt (e-mail: mrefaat_77@yahoo.co.uk).
\end{abstract}

\begin{abstract}
Keywords

- cerebellopontine angle

- epidermoid cyst

- capsule remnants

Background Epidermoid cysts (ECs)are an uncommon slowly growing congenital lesions. The cerebellopontine angle (CPA) is the most common site. Surgical management for these lesions remains challenging due to the high-adherence of the cyst capsule to the surrounding structures and its tendency to present with invasion of multiple cisterns.

Objective The aim of this study was to evaluate the clinical and radiological outcome of surgically treated ECs in the CPA region.

Materials and Methods This is a retrospective study conducted on patients presenting with symptomatic CPA ECs. Clinical outcome was assessed in terms of improving or worsening of the presenting symptoms. Radiological outcome was assessed by comparing the tumor size, brainstem, and cerebellar compression in the preoperative and postoperative images.

Results In this study, 18 patients were included. All cases were operated upon by microsurgical evacuation of the cyst contents and excision of the nonadherent parts of the capsule. On admission, cranial nerve symptoms predominated. Improvement or resolution of preoperative deficits was seen in 15 patients. Preoperative symptoms worsened in two and new postoperative symptoms occurred in one patient. Seven patients needed additional ventriculoperitoneal (VP) shunt in the same setting due to the presence of hydrocephalus. During the follow-up period, symptomatic recurrence occurred in one patient, while asymptomatic recurrence occurred in one patient.

Conclusion Surgical management of CPA ECs, leaving remnants of the capsule on cranial nerves and brain stem can be considered as a relatively safe surgical procedure with a good clinical outcome and low-recurrence risks.
\end{abstract}

\section{Introduction}

Intracranial epidermoid cysts (ECs) are congenital benign cysts with an incidence of $1 \%$ of all brain tumors. ${ }^{1}$ Almost half of ECs occur in the cerebellopontine angle (CPA), accounting for 5 to $7 \%$ of tumors in this location and the third most common brain tumor after schwannomas and meningiomas in the CPA. ${ }^{2}$ It may occur in other locations such as the suprasellar and the temporal regions. ${ }^{3}$

ECs are slowly growing and may present with symptoms of elevated intracranial pressure (ICP) or symptoms of cerebellar, brainstem, and/or cranial nerves dysfunction. ${ }^{4}$

DOI https://doi.org/ 10.1055/s-0040-1713545 ISSN 2277-954X.
MRI is the investigation of choice with diffusionweighted imaging sequence that distinguishes EC from arachnoid cyst. $^{5}$

Surgery is the best treatment choice which shows good results. Surgical treatment for these lesions appears to be difficult due to the cyst capsule's strong adherence to surrounding structures, and its ability to invade multiple cisterns. The clinical course after surgical resection of EC is however not clearly documented in the literature. ${ }^{3}$

The aim of this study was to evaluate the clinical and radiological outcomes of surgically treated EC in the CPA region. 


\section{Materials and Methods}

This study represents a retrospective review of patients surgically treated for symptomatic EC in the CPA region in the neurosurgery department of Cairo University Hospitals in the period January 2015 to December 2017.

The initial diagnosis was based on CT and MRI appearance and was histologically confirmed in all cases. Data were retrospectively collected from clinical, surgical, pathological, and radiological records.

All patients included in this study were followed-up postoperatively both clinically and radiologically for, at least, 2 years. The follow-up period ranged from 24 to 38 months. The mean follow-up period was 28.5 months.

Clinical outcome was assessed in terms of improving and worsening of the presenting symptoms. Radiological outcome was assessed by comparing the tumor size, brainstem and cerebellar compression in the preoperative and postoperative images. The results were compared with the literature.

All cases included in this study have signed our institutional consent before performing their surgeries, including their approval of participation in any future studies. The authors report no conflict of interest concerning the materials or methods used in this study or the findings specified in this paper.

\section{Results}

A total of 18 cases with CPA ECs were surgically treated in our department during the study period. Patients demography: The age of the cases ranged from 17 to 53 years, with the mean age being 38.6 years. There was a female predominance among cases that were included in this study as there were 11 females (61.1\%) and 7 males (38.9\%).

Preoperative clinical condition: all cases were symptomatic. Trigeminal neuralgia was the most common, presenting symptoms in 11 patients (61.1\%), followed by facial nerve palsy in 5 patients (27.8\%) and hearing impairment in
2 patients (11.1\%). Multiple cranial nerve affection was seen in four patients $(22.2 \%)$. Headache and blurring of vision were present in seven patients (38.9\%). Ataxia was present in five patients (27.8\%).

Surgical details: All cases were operated upon by microsurgical evacuation of the cyst contents and excision of the nonadherent parts of the capsule. Seven patients needed ventriculoperitoneal (VP) shunt in the same setting due to the presence of hydrocephalus.

Clinical outcome and complications: Improvement or resolution of preoperative symptoms was seen in 13 patients (72.2\%). Hearing impairment did not improve in two patients $(11.1 \%)$ in the follow-up period. Preoperative symptoms worsened in two patients (11.1\%). One patient presented with facial nerve palsy House-Brackman GII, which preoperatively progressed to GIV postoperatively. The other patient was presenting with partial bulbar symptoms preoperatively which increased postoperatively. New postoperative symptoms (facial nerve palsy, House-Brackman GII) occurred in one patient presenting with trigeminal neuralgia. During the follow-up period, symptomatic recurrence (trigeminal neuralgia) occurred in one patient after 26 months. No cases of postoperative chemical meningitis and/or hydrocephalus were reported in our study.

Radiological improvement regarding cerebellar and brain stem compression was seen in all patients. Asymptomatic recurrence occurred in one case in the follow up MRI images (-Figs. 1-3).

\section{Discussion}

Intracranial ECs are slowly growing, congenital anomalies occurring most commonly in the CPA. Due to the gradual mass effect, ${ }^{3}$ ECs symptomatize in a wide range of age (between 20 and 60 years) with peak occurrence at around 40 years of age..$^{5-7}$ In our study, there was a slight female predominance compared with other studies which reported similar incidence in both genders. ${ }^{5,6}$ This may be due to the smaller number of patients in our study.
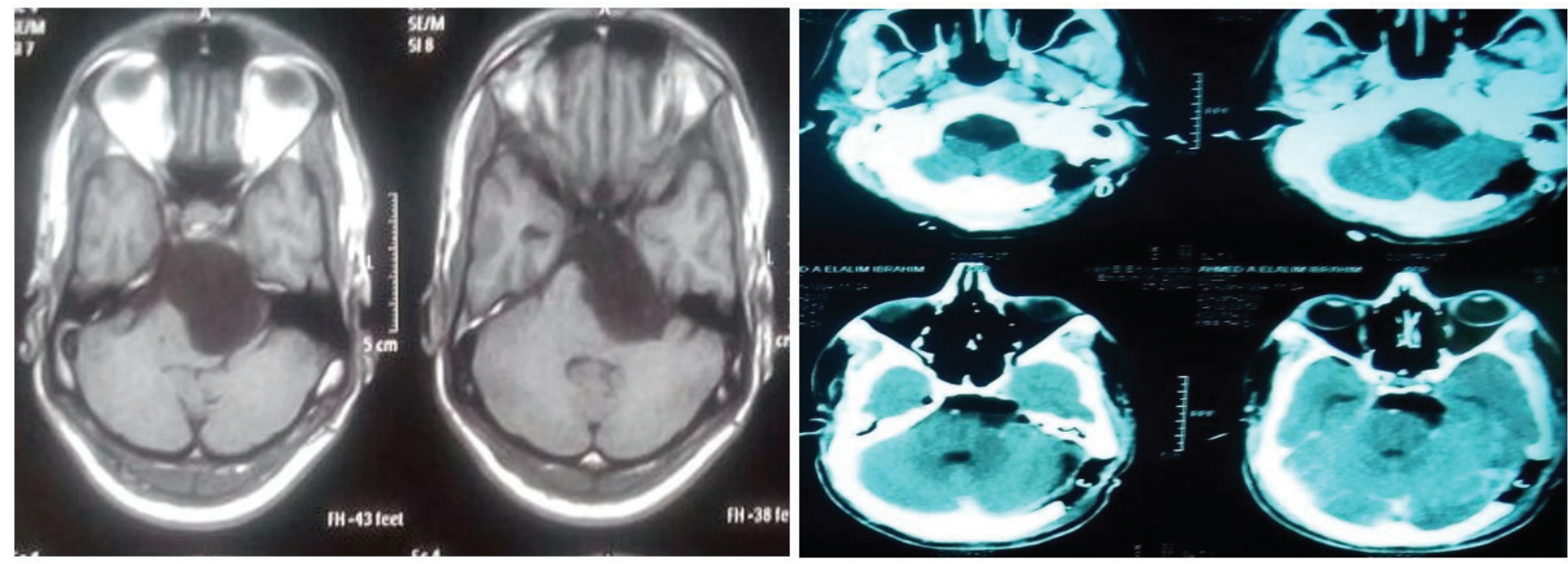

Fig. 1 Pre- and postoperative images of a 44-year-old male presenting with ataxia and multiple cranial nerve affection (trigeminal neuralgia for 4 months, facial nerve palsy [GII] and partial bulbar symptoms). Postoperative images show gross total excision of the CPA ECs with marked decrease in the preoperative brainstem and cerebellar compression. The patient had a smooth postoperative recovery with improvement of preoperative symptoms. CPA, cerebellopontine angle; EC, epidermoid cyst. 

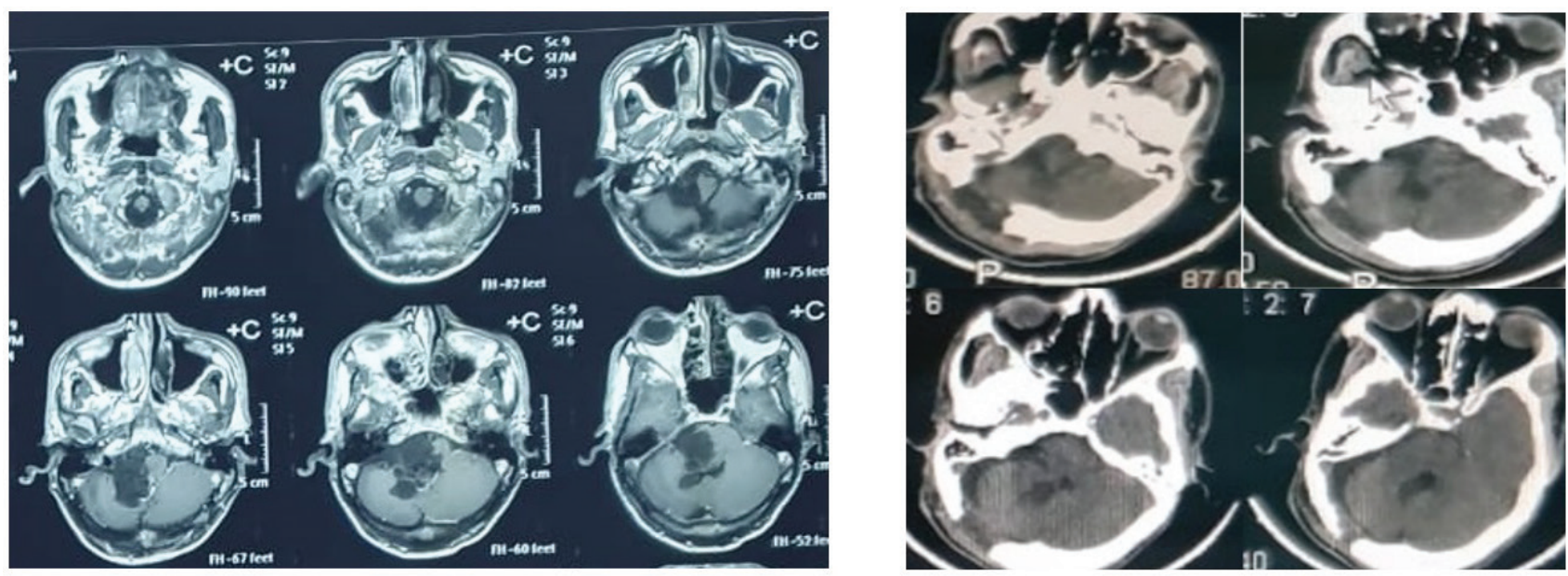

Fig. 2 Pre- and postoperative images of a 38-year-old male presenting with multiple cranial nerve affection (trigeminal neuralgia for 1 year, facial nerve palsy, and hearing impairment). Postoperative images show gross total excision of the CPA ECs. The patient had a smooth postoperative recovery with improvement of the trigeminal and facial nerves' symptoms, while he showed no improvement regarding hearing. CPA, cerebellopontine angle; EC, epidermoid cyst.
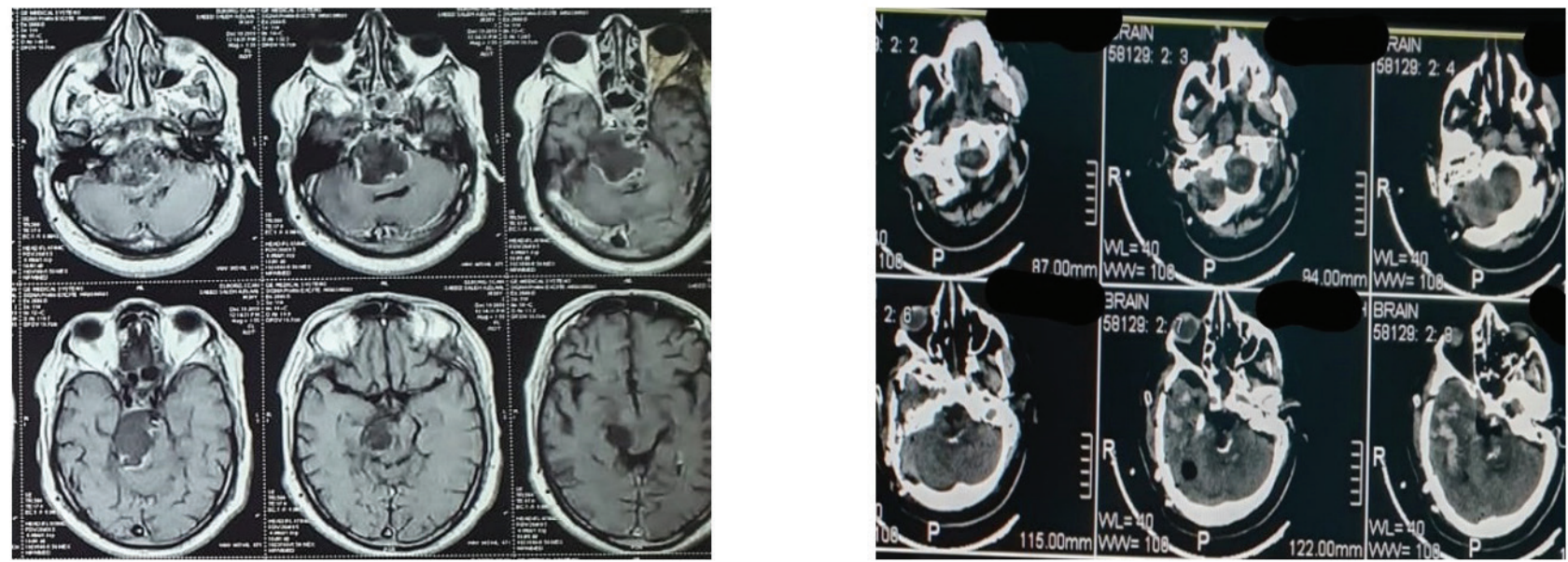

Fig. 3 Pre- and postoperative images of a 36-year-old female presenting with signs of multiple cranial nerve affection. Postoperative images show gross total excision of the CPA ECs. The patient had a smooth postoperative recovery with partial improvement of the preoperative symptoms. CPA, cerebellopontine angle; EC, epidermoid cyst.

The most common presenting symptoms are trigeminal neuralgia and hearing impairment, followed by dizziness, headaches, diplopia, and facial nerve affection. ${ }^{5,6}$ Similarly, trigeminal neuralgia was the most common presenting symptom occurring in $61 \%$ of the patients in our study group. However, facial palsy (27.8\%) was a more common symptom than hearing impairment $(11.1 \%)$ in our study.

ECs are not well-circumscribed, having irregular lobulated surfaces which usually grow and adhere to nerves and vessels or enclose them. Therefore, surgery is challenging, and incomplete excision is common due to the interaction with critical structures, as gross total excision can bring a high-risk of morbidity. ${ }^{3}$ We believe that leaving the adherent part of the capsule in place is mandatory to reduce the possibility of neurological sequelae.

Due to the linear pattern of EC growth, a symptomatic tumor recurrence may be expected after a period comparable in length to the period between birth and the time taken for the primary tumor to become symptomatic. ${ }^{8}$ Although a residue of a capsule will likely result in recurrence, this will occur many years after surgery. Berger et a ${ }^{9}$ stated that recurring symptoms can take 30 to 40 years to develop, a finding which supports the rationale for avoiding radical excision. Furthermore, several studies have found no difference in the recurrence risk between totally and incompletely excised lesions. ${ }^{6,10}$ Thus, near total excision of the tumor, leaving only tiny remnants, in a middle aged patient can be considered as clinical cure. ${ }^{2}$

Associated hydrocephalus is said to be uncommon due to the long-standing nature of the lesion. ${ }^{3}$ However, in the present study, 38.9\% had hydrocephalus and required VP shunt. Similarly, in a previously reported series, 12 out of 30 patients (40\%) required shunt surgery for hydrocephalus. ${ }^{11}$

The temporary worsening of symptoms is likely due to nerve manipulation or partial damage during surgery. ${ }^{3,10,12}$ In the previously reported studies, a relatively 
high-incidence of postoperative cranial nerve dysfunction was identified which appeared to recover during the follow-up period in most cases. ${ }^{9,13,14}$ In our study, there was a low-incidence of cranial nerve dysfunction postoperatively, since the adherent tumor capsule was left behind. This was observed in two (11.1\%) of our patients, where one patient progressed to House-Brackman GIV facial palsy from GII preoperatively, which gradually improved in the follow-up period. The other patient was presenting with partial bulbar symptoms preoperatively which increased postoperatively. New postoperative symptoms in the form of facial nerve palsy House-Brackman GII occurred in one patient presenting with trigeminal neuralgia which rapidly recovered.

The postoperative complication rate in our series was $16.6 \%$ with no mortalities. Close values have been reported previously., ${ }^{3,15}$ A particular clinical complication of EC is aseptic meningitis, which is due to the spill of cholesterol crystals into the cerebrospinal pathway. ${ }^{11,13,17}$ The incidence of aseptic meningitis ranges from 0 to $18.2 \%{ }^{10,12}$ Continuous intraoperative CPA cistern irrigation with hydrocortisone solution and delayed postoperative steroids withdrawal were recommended as potential measures to avoid chemical meningitis. ${ }^{9,11,17}$ In the present study, the same protocol was implemented in all patients and this complication was not reported.

Communicating hydrocephalus may develop after meningitis or following cyst content leakage from the surgical field. ${ }^{9,11,18}$ There was no postoperative hydrocephalus in our series which needed CSF diversion. We assume that regular perioperative corticosteroid administration and surgical field irrigation with hydrocortisone solution and saline reduces the risk of postoperative hydrocephalus development.

Follow-up MRIs are needed for recurrence assessment. 5,6,12 Most authors agree that surgery is needed in the event of a deterioration of a chronic neurological disability or the emergence of a new impairment due to tumor growth and not simply because of the detection of tumor regrowth in imaging studies., ${ }^{2,6,12}$ In the present study, radiological improvement regarding cerebellar and brain stem compression was seen in all patients. Radiological recurrence occurred in two (11.1\%) patients in the follow-up period. Symptomatic recurrence (trigeminal neuralgia) occurred in one patient after 26 months and required reoperation. Asymptomatic recurrence occurred in the other patient, which was detected in the follow up MRI 32 months after surgery, and no surgery was done to this patient, as surgery is often indicated only when the recurrent lesion is symptomatic. ${ }^{18}$

\section{Conclusion}

Surgical management of EC in the CPA region, leaving remnants of the capsule on blood vessels, nerves, and brain stem can be considered as a relatively safe surgical procedure with a good clinical outcome and low-recurrence risks.

\section{Note}

This study was previously presented at 42nd Egyptian Congress of Neurosurgery held in Cairo, Egypt from 27th to 29th March 2019.

\section{Conflict of Interest}

None declared.

\section{References}

1 Chen CY, Wong JS, Hsieh SC, Chu JS, Chan WP. Intracranial epidermoid cyst with hemorrhage: MR imaging findings. Am J Neuroradiol 2006;27(2):427-429

2 Czernicki T, Kunert P, Nowak A, Wojciechowski J, Marchel A. Epidermoid cysts of the cerebellopontine angle: Clinical features and treatment outcomes. Neurol Neurochir Pol 2016;50(2):75-82

3 Rehman L, Bokhari I, Siddiqi SU, Bagga V, Hussain MM. Intracranial epidermoid lesions: our experience of 38 cases. Turk Neurosurg 2017

4 Hasegawa M, Nouri M, Nagahisa S, et al. Cerebellopontine angle epidermoid cysts: clinical presentations and surgical outcome. Neurosurg Rev 2016;39(2):259-266

5 Kato K, Ujiie H, Higa T, et al. Clinical presentation of intracranial epidermoids: a surgical series of 20 initial and four recurred cases. Asian J Neurosurg 2010;5(1):32-40

6 Schiefer TK, Link MJ. Epidermoids of the cerebellopontine angle: a 20-year experience. Surg Neurol 2008;70(6):584-590

7 Alemdar M. Epidermoid cyst causing hemifacial spasm epidermoid cyst in cerebellopontine angle presenting with hemifacial spasm. J Neurosci Rural Pract 2012;3(3):344-346

8 Nagasawa D, Yew A, Safaee M, et al. Clinical characteristics and diagnostic imaging of epidermoid tumors. J Clin Neurosci 2011;18(9):1158-1162

9 Berger MS, Wilson CB. Epidermoid cysts of the posterior fossa. J Neurosurg 1985;62(2):214-219

10 Kobata H, Kondo A, Iwasaki K. Cerebellopontine angle epidermoids presenting with cranial nerve hyperactive dysfunction: pathogenesis and long-term surgical results in 30 patients. Neurosurgery 2002;50(2):276-285

11 Mohanty A, Venkatrama SK, Rao BR, Chandramouli BA, Jayakumar PN, Das BS. Experience with cerebellopontine angle epidermoids. Neurosurgery 1997;40(1):24-29

12 Safavi-Abbasi S, Di Rocco F, Bambakidis N, et al. Has management of epidermoid tumors of the cerebellopontine angle improved? A surgical synopsis of the past and present. Skull Base 2008;18(2):85-98

13 Vinchon M, Pertuzon B, Lejeune JP, Assaker R, Pruvo JP, Christiaens JL. Intradural epidermoid cysts of the cerebellopontine angle: diagnosis and surgery. Neurosurgery 1995;36(1):52-56

14 Yaşargil MG, Abernathey CD, Sarioglu AC. Microneurosurgical treatment of intracranial dermoid and epidermoid tumors. Neurosurgery 1989;24(4):561-567

15 Ali M, Khanzada K, Khan H, et al. Cranial dermoid and epidermoid tumors. J Postgrad Med Inst 2008;22(1):33-36

16 Lopes M, Capelle L, Duffau H, et al. [Surgery of intracranial epidermoid cysts. Report of 44 patients and review of the literature]. Neurochirurgie 2002;48(1):5-13

17 Samii M, Tatagiba M, Piquer J, Carvalho GA. Surgical treatment of epidermoid cysts of the cerebellopontine angle. J Neurosurg 1996;84(1):14-19

18 Sabin HI, Bordi LT, Symon L. Epidermoid cysts and cholesterol granulomas centered on the posterior fossa: twenty years of diagnosis and management. Neurosurgery 1987; 21(6):798-805 